\title{
SALIVARY LEVEL OF INTERLEUKIN-17 IN PATIENTS HAVING ATROPHIC AND EROSIVE ORAL LICHEN PLANUS BEFORE AND AFTER TREATMENT WITH TOPICAL STEROIDS (A CONTROLLED CLINICAL TRIAL)
}

\author{
Ahmed Abdel Aziz Hassan*, Mai Talaat Elgendi**, Olfat Shaker*** and Radwa. R. Hussein ${ }^{* * * *}$
}

\begin{abstract}
Background: The present study was aimed to measure the salivary level of IL-17 in patients having atrophic and erosive Oral lichen planus lesions (OLP) and to evaluate the effect of topical corticosteroids used for treatment of erosive/atrophic oral lichen planus on the level of IL-17 in the saliva.
\end{abstract}

Subjects and methods: The present study was performed on 40 subjects divided into 3 groups Group (I) Twenty patients diagnosed with erosive/atrophic OLP received topical steroid (triamcinolone acetonide $0.1 \%$ ) four times per day for four weeks. Group (II) Ten patients diagnosed with reticular OLP and Group (III) Ten patients with normal mucosa (healthy control Group). Salivary samples were collected before and after treatment in group (I) and once from subjects in group (II) and (III) for determination of IL-17 levels using enzyme-linked immunosorbent assay (ELISA).

Results: The mean IL-17 levels in saliva of erosive/atrophic OLP group were statistically significantly higher before treatment with topical steroid than after treatment and both are statistically significantly higher than the reticular group and healthy control subjects. Also, the clinical score and pain visual analogue score values were statistically significantly higher in the erosive/atrophic group before treatment than after treatment.

Conclusions: The salivary levels of IL-17 in erosive/atrophic OLP patients was higher than the reticular OLP patients and both are higher than the healthy control subjects, suggesting that IL-17 has a major role in the pathogenesis of OLP and its salivary level may be diagnostic for the severity of the condition of the erosive/atrophic OLP.

KEYWORDS: IL-17, Oral lichen planus, saliva.

\footnotetext{
* Assistant Professor of Oral Medicine, Periodontology and Diagnosis, Faculty of Dentistry, Ain Shams University, Egypt ** Assistant Lecturer of Oral Medicine, Periodontology and Diagnosis, Faculty of Dentistry, Future University., MSc Oral Medicine, Ain Shams University Egypt.

*** Professor of Medical Biochemistry, Faculty of Medicine, Cairo University Egypt.

**** Lecturer of Oral Medicine, Periodontology and Diagnosis, Faculty of Dentistry, Ain Shamus University, Egypt.
} 


\section{INTRODUCTION}

In 2005 T helper 17 (Th17) cells were identified as a new subset of CD4+ $\mathrm{T}$ helper cells producing interleukin-17 (IL-17). In response to environmental signs, naive $\mathrm{T}$ cells differentiate into Th17 effector cells. Interleukin-17 and other Th17 cytokines are related to the pathogenesis of diverse autoimmune and inflammatory diseases. (Harrington et al., 2005; Park et al., 2005).

Oral lichen planus (OLP) is considered one of mucocutaneous immune mediated diseases which affects the oral mucosa. The clinical presentation of the oral lesions is divided into six clinical forms which are: papular, reticular, plaque-like lesions, atrophic, erosive / ulcerative and bullous erosive. The papular, reticular and plaque like are asymptomatic types. Oppositely, atrophic, erosive/ulcerative and bullous lesions are the symptomatic types with symptoms ranging from soreness to severe burning pain (Eltohami et al. 2018).

The etiology of OLP is not completely understood. A lot of factors have been suggested for the etiology including genetic background, infectious agents (bacterial and viral infections), autoimmunity-associated with other autoimmune diseases, immunodeficiency, stress, trauma and systemic conditions like diabetes \& hypertension. (Nosratzehi, et al., 2018; Mutafchieva et al. 2018).

IL-17 is a critical cytokine in the immune pathogenesis of LP. Under inflammatory conditions, it induces chemokine release from various cell types of the skin including: endothelial cells, macrophages, and keratinocytes leading to tissue remodeling and the recruitment of pro-inflammatory effector cells in the skin (Hirota et al., 2011).

In 2012, Shaker and Hassan investigated the role of IL-17 in OLP pathogenesis. IL-17 serum levels were found markedly higher in OLP patients compared with healthy volunteers. They concluded that IL-17 can contribute to OLP pathogenesis by $\mathrm{T}$ cell mediated reactions enhancement and encouraging release of cytokines. Another study was done by Xie et al., they compared serum concentrations of IL-17 in healthy people and patients with erosive/atrophic and reticular types of OLP. They found that the serum IL-17 concentrations were significantly higher in patients with erosive/ atrophic OLP than the reticular form and both are higher than the control group. (Xie et al., 2012).

In 2015 Wang et al., conducted a study to assess the levels of IL-17 in saliva in patients having reticular and erosive/atrophic types of OLP and healthy volunteers and they concluded that Salivary IL-17 concentrations in erosive OLP group were significantly higher than in those with reticular OLP and in healthy controls. Moreover, they found a positive correlation were between IL-17 salivary concentrations and disease clinical scores (CS) (Wang et al., 2015).

Topical corticosteroid is the most useful drug for OLP treatment. It has strong anti-inflammatory and immune-modulatory action, in addition to the reduction in the number and function of several immune cells such as, $\mathrm{T}$ and $\mathrm{B}$ lymphocytes, monocytes, neutrophils, and eosinophils at sites of inflammation. It decreases the production of cytokines, chemokines and boosts the production of macrophage migration inhibitory factor (Thongprasom \& Dhanuthai, 2008).

\section{AIM OF THE STUDY}

To measure the level of IL-17 in the saliva of patients having atrophic and erosive oral lichen planus lesions as a primary outcome and to evaluate the effect of topical corticosteroids used for treatment of oral lichen planus on the level of IL-17 in the saliva. 


\section{MATERIAL AND METHODS}

Forty subjects were included in this study, twenty of them were proven to have clinically and histologically erosive-atrophic OLP, ten subjects were proven clinically and histologically having reticular type of OLP and ten healthy control subjects having no oral or skin lesions of LP were assessed for their eligibility for this study. All patients and the healthy subjects were selected from the out-patient clinic of Oral Medicine, Periodontology, Oral Diagnosis and Radiology department Faculty of Dentistry Ain Shams University.

\section{Inclusion criteria:}

Participants were from both genders, aged from 25 to 60 years. They were systemically free according to the American Society of Anesthesiologists classification (ASA I). Furthermore, they were selected to be free of any oral lesions other than OLP. No history of previous treatments potentially effective on OLP (Topical treatment from two weeks or systemic steroid from four weeks) (Swift et al., 2005).

\section{Exclusion criteria:}

Smokers, Pregnant or breast-feeding females, vulnerable group of patients (prisoners, handicapped and mentally retarded individuals) were excluded from this study.

\section{Ethical procedures:}

Each patient was provided with detailed verbal and written information on the study protocol. Understanding and agreement to enroll in the study was confirmed and a written informed consent was obtained. The study was approved by the ethical committee of faculty of dentistry Ain Shams University and took the number FDAsuRecIM021824.

The forty selected participants were assigned into three groups as follows:

Group I Included twenty patients having atrophic and erosive oral lichen planus and received topical steroid (triamcinolone acetonide $0.1 \%$ ) * four times per day (after each meal and at bed time) for four weeks

Group II Included ten patients suffering from reticular oral lichen planus as a positive control group.

Group III Included ten healthy volunteers having no oral lichen planus as a negative control group.

Sampling: The un-stimulated whole saliva (UWS) was collected between 10:00 a.m. and 12:00 p.m., and at least 90 minutes after the last intake of drink or food. It is obtained by requesting subjects to swallow first then tilt head forward, saliva was collected by aspiration using sterile syringe and immediately stored in Eppendorf tubes, and they left to freeze at or below $-20^{\circ} \mathrm{C}$ to prevent bacterial growth and further degradation of salivary samples. IL-17 levels were assessed by using Human IL-17 (ELISA) kits provided from BMS2017/BMS2017TEN supplied from eBioscience, Affymetrix (Vienna, Austria).

The clinical score values (CS) and pain visual analogue scale (P-VAS) were measured before and throughout the treatment period in group I and it was measured at the baseline at group II \& group III .The salivary samples were taken before and after treatment period in group I (4 weeks), while the salivary samples in group II and III were taken only once at baseline with no treatment.

\footnotetext{
* GEO-ORALOG (Triamcinolone Acetonide 0.1\%)

$15 \mathrm{mg}$ Oral past, Marcyrl pharmaceutical industries. El Obour city the west extension block 20005, Egypt
} 


\section{RESULTS}

The results of the present study showed that the mean and SD values of salivary IL-17 concentrations in group (I) before treatment was $(50.65 \pm 2.29$ $\mathrm{pg} . / \mathrm{ml})$ and it was $(41.86 \pm 3.25 \mathrm{pg}, / \mathrm{ml})$ after treatment with an extremely statistically significant difference before and after treatment $(\mathrm{p}<0.001)$ and the percentage change was $(17.35 \pm 5.34 \%)$.

For group (II), the mean and SD values of IL17 salivary concentration was $(39.24 \pm 1.56 \mathrm{pg} / \mathrm{ml})$ while for group (III) it was $(33.51 \pm 0.83 \mathrm{pg} / \mathrm{ml})$. There was an extremely statistically significant difference between the mean and SD values of IL-17 levels in saliva between group (I) before treatment and both group (II) and group (III) and between group (II) and group (III) $(\mathrm{p}<0.001)$. Also, there was an extremely statistically significant difference between the mean and SD values of IL-17 levels in saliva between group (I) after treatment and both group (II) and group (III) $(\mathrm{p}<0.001)$.

The (CS) of group I before treatment was $(4.35 \pm 0.67)$ and after treatment it was $(2.55 \pm 0.60)$ and the percentage change was $(41.37 \pm 30.53 \%)$ and there was an extremely statistically significant difference in the mean and SD values of the CS before and after treatment $(p<0.001)$.
The mean and SD value for (P-VAS) at baseline for group I was (7.25 \pm 0.90$)$, after 1 week of treatment it was $(6.45 \pm 0.92)$, after 2 weeks it was $(3.75 \pm 1.21)$, after 3 weeks it was $(1.05 \pm 1.19)$, and after 4 weeks it was $(1.20 \pm 1.11)$, the percentage change between baseline and 1 week it was $(11.11 \pm 4.88 \%)$, between 1 week and 2 weeks it was $(42.50 \pm 13.75 \%)$, between 2 weeks and 3 weeks it was $(78.00 \pm 23.92 \%)$, between 3 weeks and 4 weeks it was $(0.00 \pm 0.00 \%)$ and between baseline and 4 weeks it was $(84.53 \pm 13.81 \%)$. There was an extremely statistically significant difference between baseline and two weeks, baseline and three weeks, baseline and after 4 weeks $(p<0.001)$. Also, there was an extremely statistically significant difference between one week and three weeks, one week and 4 weeks, and there was an extremely statistically significant difference between two weeks and three weeks, two weeks and 4 weeks $(p<0.001)$. There was a strong positive correlation between CS values and IL-17 salivary concentration levels before and after 4 weeks of treatment in group I and this correlation was strongly statistically significant $(\mathrm{rs}=0.826)(\mathrm{p}<0.001)$. There was also a strong positive correlation between P-VAS and IL17 salivary concentration levels between baseline and after 4 weeks of treatment and this correlation was extremely statistically significant $(r s=0.868)$ $(\mathrm{p}<0.001)$.

TABLE (1) The mean and (SD) values for IL-17 salivary concentration in the three study groups

\begin{tabular}{|c|c|c|c|c|c|}
\hline \multirow{2}{*}{ Group } & \multicolumn{2}{|c|}{ Group I } & \multirow{2}{*}{ Group II } & Group III & P-value \\
\cline { 2 - 3 } & Before treatment & After treatment & & \\
\hline IL-17 & $50.65 \pm 2.29^{\mathrm{A}}$ & $41.86 \pm 3.25^{\mathrm{B}}$ & $39.24 \pm 1.56^{\mathrm{C}}$ & $33.51 \pm 0.83^{\mathrm{D}}$ & $<\mathbf{0 . 0 0 1 *}$ \\
\hline
\end{tabular}

TABLE (2) Mean and (SD) values for (CS) in Group I before \& after treatment

\begin{tabular}{|c|c|c|c|}
\hline \multirow{2}{*}{} & \multicolumn{2}{|c|}{$(\mathrm{CS})($ Mean \pm SD) } & \multirow{2}{*}{ P-value } \\
\cline { 2 - 3 } & Before treatment & After treatment & \\
\hline Group One & $4.35 \pm 0.67$ & $2.55 \pm 0.60$ & $<\mathbf{0 . 0 0 1 *}$ \\
\hline
\end{tabular}


TABLE (3) Mean and (SD) values for (P-VAS) in group I

\begin{tabular}{|c|c|c|c|c|c|c|}
\hline \multirow{2}{*}{ Groups } & \multicolumn{5}{|c|}{ (P-VAS) } & \multirow{2}{*}{ p-value } \\
\cline { 2 - 7 } & Baseline & $1^{\text {st }}$ weeks & $2^{\text {nd }}$ weeks & $3^{\text {rd }}$ weeks & 4 weeks & \\
\hline Group (I) & $7.25 \pm 0.90^{\mathrm{A}}$ & $6.45 \pm 0.92^{\mathrm{AB}}$ & $3.75 \pm 1.21^{\mathrm{B}}$ & $1.05 \pm 1.19^{\mathrm{C}}$ & $1.20 \pm 1.11^{\mathrm{C}}$ & $<0.001^{*}$ \\
\hline
\end{tabular}

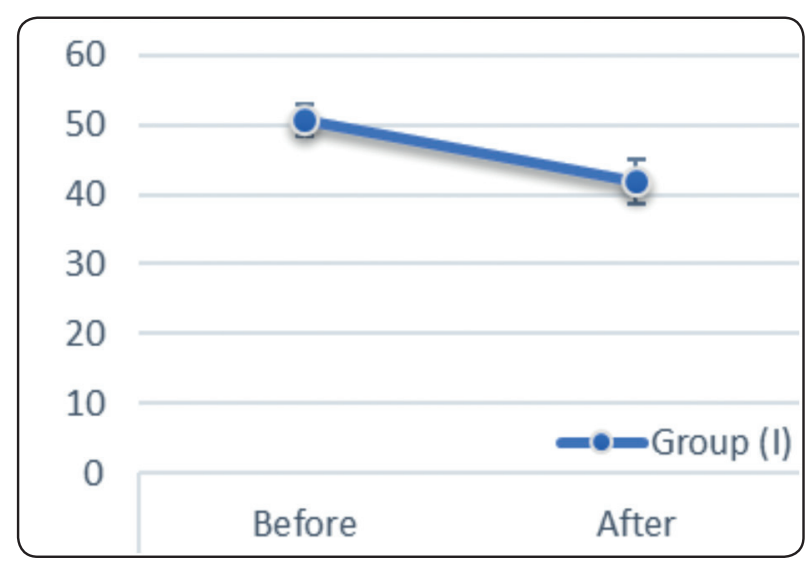

Line chart (1) Showing mean and (SD) values of IL-17 salivary concentration $(\mathrm{pg} / \mathrm{ml})$ in group (I) before and after treatment.

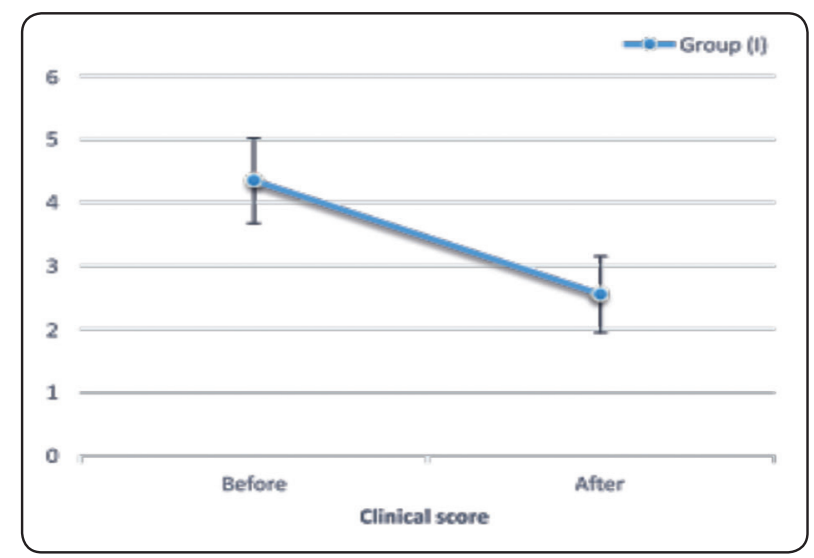

Line chart (2) Mean and (SD) values for (CS) in Group I before $\&$ after treatment

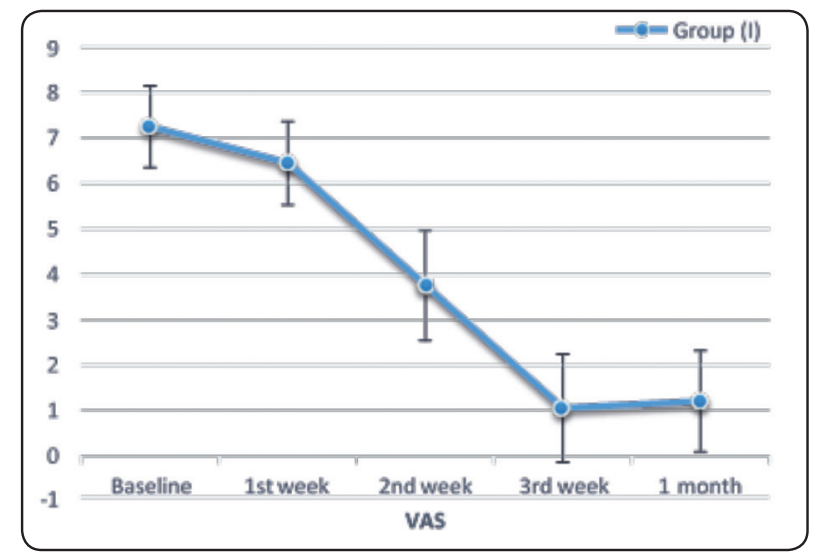

Line charting (3) Mean and (SD) values for (P-VAS) in group I.

\section{DISCUSSION}

In the present study the salivary IL-17 levels were assessed and there was an extreme statistically significant difference between the mean and standard deviation values of IL-17 levels between group (I) and group (II), between group (I) and group (III), and there was a statistically significant difference between group (II) and group (III). These results were in accordance with the results of the study conducted by Wang et al where they found that there was a statistically significant difference between the salivary IL-17 levels in a erosive/ atrophic group and the reticular group, and between the erosive group and the healthy control group, while unlike the results of the present study there was no statistical significant difference between the reticular group and the healthy control one (Wang et al., 2015).

The results of present study were in accordance with the results of the study of El-Refai et al., where they found that there was a statistically significant difference in the mean values of IL-17 levels in saliva between the erosive/atrophic OLP group and both the PV group and the control subjects $(\mathrm{p}=$ $0.03)$. The salivary levels of IL-17 in erosive OLP in their study was $(4.38 \pm 0.44 \mathrm{pg} / \mathrm{ml})$ which is much lower than the salivary levels of IL-17 in the present study and this may be due to they centrifuged the salivary samples (2000g for $10 \mathrm{~min}$ ), and stored the supernatants at $-20^{\circ} \mathrm{C}$ to be used for analysis and the supernatant may contain low levels of IL-17, while in the present study the whole salivary sample was stored at $-20{ }^{\circ} \mathrm{C}$ to be used for analysis (El-Refai et al., 2019). 
In the present study, the (CS) was measured in group I before treatment and after treatment and there was an extreme statistically significant difference between the mean and standard deviation values of the clinical score $(\mathrm{p}<0.001)$.These results were in accordance with the results of Siponen et $\boldsymbol{a l}$. who used topical steroid (triamcinolone acetonide) for the treatment of erosive /atrophic OLP and found that there was a statistically significant difference between the mean and standard deviation values of the (CS) before and after treatment. The (CS) in their study before treatment was much higher than the clinical score in our study before treatment, and the (CS) in their study after treatment also was higher than the (CS) values after treatment in our study. But the improvement in their study was almost one third the (CS) value, while the improvement in our study was $41 \%$ of the (CS) value (Siponen et al., 2017).

In the present study the (P-VAS) was measured in group I and found that there was an extreme statistically significant difference between baseline and 2 weeks, baseline and 3 weeks, between baseline and after 4 weeks. There was also an extreme statistically significant difference between 1 week and 3 weeks, 1 week and 4 weeks, and there was an extreme statistically significant difference between 2 weeks and 3 weeks, 2 weeks and 4 weeks $(\mathrm{p}<0.001)$.

The results of the present study regarding the (P-VAS) were in accordance with Suzan and Hadir, where they found that there was an extreme statistically significant difference between baseline and 2 weeks after treatment, baseline and 1 month after treatment and between baseline and 2 months after treatment. There was an extreme statistical difference between 2 weeks and 1 month, 2 weeks and 2 months and between 1 month and 2 months after treatment $(\mathrm{p}<0.001)($ Suzan \& Hadir, 2012).

In the present study there was a strong positive correlation between the (CS) and IL-17 salivary concentration values before and after treatment in the erosive/atrophic form of OLP which was statistically significant $(\mathrm{p}<0.001)$. Also, there was a strong positive correlation between (P-VAS) and IL17 salivary values before and after treatment which was statistically significant $(\mathrm{p}<0.001)$. These results were in accordance with Wang et al. where they studied the salivary IL-17 levels in patients having OLP and found a positive correlation between IL-17 levels in saliva and the (CS)of the erosive/atrophic OLP patients ( $\mathrm{P}=0.003)$ (Wang et al., 2015).

\section{CONCLUSION}

The results of the present study demonstrated that the levels of IL-17 in saliva are higher in the erosive/atrophic types of OLP than the reticular types and both are higher than the healthy control subjects. Also, the levels of IL-17 in saliva in patients having erosive/atrophic OLP decreased with the use of topical triamcinolone acetonide $0.1 \%$ and there was a positive correlation between the (CS) and the levels of IL-17 in erosive/atrophic group. There was also a positive correlation between the (P-VAS) scale and the levels of IL-17 in erosive/ atrophic group. All these data suggested that IL-17 has a major role in the pathogenesis of OLP and its salivary level may be of diagnostic value for the severity of the condition of the erosive/atrophic OLP.

\section{REFERENCES}

1. El-Refai I, Maged A, \& El-Saady D (2019). Assessment of IL-17 in Oral Lichen Planus and in Pemphigus Vulgaris. Egyptian Dental Journal, 65, 343-350.

2. Eltohami YI, Alim NE \& Abuaffan AH (2018). "Sudanese male with erosive lichen planus case." Journal of Hospital Medicine Management. 2:2.

3. Harrington LE, Hatton RD, Mangan PR, Turner H, Murphy TL \& Murphy KM (2005). Interleukin 17-producing $\mathrm{CD}^{+}{ }^{+}$effector $\mathrm{T}$ cells develop via a lineage distinct from the $\mathrm{T}$ helper type 1 and 2 lineages. Nature Immunology; $6: 1123-32$

4. Hirota K, Duarte JH, Veldhoen M, Hornsby E \& Cua DJ (2011). Fate mapping of IL-17-producing $\mathrm{T}$ cells in inflammatory responses. Nature Immunology; 12:255-63. 10.1038/ni.1993 
5. Mutafchieva MZ, Draganova-Filipova MN, Zagorchev PI \& Tomov GT (2018). Oral lichen planus-known and unknown: a review. Folia medica, 60(4), pp.528-535.

6. Nosratzehi T (2018). Oral lichen planus: an overview of potential risk factors, biomarkers and treatments. Asian Pacific journal of cancer prevention: APJCP, 19(5), p.1161.

7. O'Quinn D, Palmer M, Lee Y\& Weaver C (2008). Emergence of the Th17 pathway and its role in host defense. Advances in Immunology; 99:115-63.

8. Park H, Li Z, Yang XO, Chang SH, Nurieva R, Wang YH \& Dong C (2005). A distinct lineage of CD4 T cells regulates tissue inflammation by producing interleukin 17 . Nature immunology, 6(11), 1133-1141.

9. Shaker O \& Hassan AS (2012). Possible role of interleukin-17 in the pathogenesis of lichen planus. British Journal of Dermatology; 166:1367-8.
10. Siponen M, Huuskonen L, Kallio-Pulkkinen S, Nieminen P \& Salo T (2017). Topical tacrolimus, triamcinolone acetonide, and placebo in oral lichen planus: a pilot randomized controlled trial. Oral Diseases, 23(5), 660-668.

11. Suzan SI \& Hadir FED (2012). Topical pimecrolimus versus Triamcinolone acetonide paste in the treatment of oral lichen planus. Egyptian dental journal, 58, 1.

12. Swift JC, Rees TD, Plemons JM, Hallmon WW\& Wright JC (2005). The effectiveness of $1 \%$ pimecrolimus cream in the treatment of oral erosive lichen planus. Journal of periodontology, 76(4), 627-635.

13. Thongprasom K \& Dhanuthai K (2008). Steroids in the treatment of lichen planus: a review. Journal of oral science, 50(4), 377-385.

14. Wang K, Miao T, Lu W, He J, Cui B, Li J \& Xiao L (2015). Analysis of oral microbial community and Th17associated cytokines in saliva of patients with oral lichen planus. Microbiology and immunology, 59(3), 105-113. 